PROCEEDINGS OF THE

AMERICAN MATHEMATICAL SOCIETY

Volume 140, Number 2, February 2012, Pages 393-402

S 0002-9939(2011)10905-5

Article electronically published on June 1, 2011

\title{
GONALITY AND CLIFFORD INDEX OF PROJECTIVE CURVES ON RULED SURFACES
}

\author{
YOUNGOOK CHOI AND SEONJA KIM
}

(Communicated by Bernd Ulrich)

\begin{abstract}
Let $X$ be a smooth curve on a ruled surface $\pi: S \rightarrow C$. In this paper, we deal with the questions on the gonality and the Clifford index of $X$ and on the composedness of line bundles on $X$ with the covering morphism $\left.\pi\right|_{X}$. The main theorem shows that if a smooth curve $X \sim a C_{o}+\mathbf{b} f$ satisfies some conditions on the degree of $\mathbf{b}$, then a line bundle $\mathcal{L}$ on $X$ with $\operatorname{Cliff}(\mathcal{L}) \leq$ $a g(C)-1$ is composed with $\left.\pi\right|_{X}$. This implies that a part of the gonality sequence of $X$ is computed by the gonality sequence of $C$ as follows:
\end{abstract}

$$
d_{r}(X)=a d_{r}(C) \text { for } r \leq L,
$$

where $L$ is the length of the gonality sequence of $C$.

\section{INTRODUCTION}

Throughout this paper, $X$ is a smooth irreducible algebraic curve of genus $g$ over an algebraically closed field of characteristic 0 . If $X$ is a multiple covering of a smooth irreducible curve $C$, it is natural to raise a question on the relations between $X$ and $C$ with respect to the gonality and the Clifford index, which are important birational numerical invariants for algebraic curves. Here, the Clifford index $\operatorname{Cliff}(X)$ and the gonality gon $(X)$ of a smooth curve $X$ are defined by

$$
\begin{gathered}
\operatorname{gon}(X):=\min \left\{n \mid \text { there is a } g_{n}^{1} \text { on } X\right\}, \\
\operatorname{Cliff}(X):=\min \left\{\operatorname{Cliff}(\mathcal{L}) \mid h^{0}(X, \mathcal{L}) \geq 2, h^{1}(X, \mathcal{L}) \geq 2\right\},
\end{gathered}
$$

where $\operatorname{Cliff}(\mathcal{L})=\operatorname{deg} \mathcal{L}-2 h^{0}(X, \mathcal{L})+2$ for a line bundle $\mathcal{L}$ on $X$. It is known that $2 \leq \operatorname{gon}(X) \leq\left[\frac{g+3}{2}\right]$ by Meis' Theorem $([13])$ and $\operatorname{gon}(X)-3 \leq \operatorname{Cliff}(X) \leq$ $\operatorname{gon}(X)-2$ by the Theorem in 3 .

An expectation on the gonality and the Clifford index of $X$ is

$$
\operatorname{gon}(X)=(\operatorname{deg} \phi) \times \operatorname{gon}(C) \text { and } \operatorname{Cliff}(X)=(\operatorname{deg} \phi) \times \operatorname{gon}(C)-2 .
$$

Received by the editors September 28, 2009 and, in revised form, November 16, 2010.

2010 Mathematics Subject Classification. Primary 14H51, 14J26, 14H45.

Key words and phrases. Gonality, Clifford index, ruled surface, multiple covering, CastelnuovoSeveri inequality, gonality sequence.

The first author's work was supported by the Korea Research Foundation Grant funded by the Korean Government (KRF-2008-314-C00011).

The second author's work was supported by the Basic Science Research Program through the National Research Foundation of Korea (NRF), funded by the Ministry of Education, Science and Technology (2009-0075469).

(C)2011 American Mathematical Society Reverts to public domain 28 years from publication 
We can also have a further question on the degree range in which any base point free line bundles on $X$ are composed with the covering morphism $\phi: X \rightarrow C$. Here, a line bundle $\mathcal{L}$ on $X$ is said to be composed with the covering morphism $\phi$ if $\mathcal{L}$ is a pull-back of a line bundle on $C$ via $\phi$. Note that the morphism associated to the base point free part of such a line bundle factors through the covering morphism $\phi$.

The typical well-known cases in these directions are hyperelliptic curves and general $k$-gonal curves: The base point free part of a special line bundle $\mathcal{L}$ on a hyperelliptic curve $X$ with $\operatorname{deg} \mathcal{L} \leq 2 g-2$ is composed with the covering morphism $\phi: X \stackrel{2: 1}{\longrightarrow} \mathbb{P}^{1}$. For a general $k$-gonal curve $X$, it is also known that a base point free line bundle $\mathcal{L}$ with $\operatorname{Cliff}(\mathcal{L}) \leq\left[\frac{g-4}{2}\right]$ and $\operatorname{deg} \mathcal{L} \leq g-1$ is composed with the covering morphism $\phi: X \stackrel{k: 1}{\longrightarrow} \mathbb{P}^{1}([9])$.

In these cases, not only is the base curve the simplest curve $\mathbb{P}^{1}$, but the covering morphism is also simple; i.e., it does not factor through a non-trivial morphism. In fact, if the covering morphism is simple, then we can apply the CastelnuovoSeveri inequality: it bounds the degree of line bundles which are not composed with the the covering morphism. Thus, in the cases where the covering morphisms are simple, there are some theorems to determine whether a given line bundle is or is not composed with $\phi(2$, 11], 15, [10]).

On the other hand, if a covering morphism is not simple, it is hard to determine whether or not given line bundles are composed. But if a covering $X$ of a smooth curve $C$ is embedded in a ruled surface $S$ with the natural projection $\pi: S \rightarrow C$, one can use known properties on the surface $S$ and the base curve $C$ to show the composedness of line bundles on $X$ with its covering morphism $\left.\pi\right|_{X}$. G. Martens and T. Harui have shown in [12] and [6] that the expectation (*) on the gonality and Clifford index holds for smooth curves on Hirzeburch surfaces and elliptic ruled surfaces, respectively.

In this paper, we deal with the gonality and the Clifford index of $X$ and with the composedness of line bundles on $X$ with $\left.\pi\right|_{X}$ for a smooth curve $X$ on ruled surfaces $\pi: S \rightarrow C$. First of all, we show that such a covering $\left.\pi\right|_{X}$ is not always simple (see Example 2.2 and Example 2.3). The main theorem (Theorem 3.10) shows that if a smooth curve $X \sim a C_{o}+\mathbf{b} f$ on a ruled surface $\pi: S \rightarrow C$ satisfies some conditions on the degree of $\mathbf{b}$, then a line bundle $\mathcal{L}$ on $X$ with $\operatorname{Cliff}(\mathcal{L}) \leq a g(C)-1$ is composed with $\left.\pi\right|_{X}$. For $g(C) \geq 4$, this is a stronger result than the expectation (*) on the gonality and Clifford index: Under the same conditions of the main theorem, we obtain a result on the gonality sequence of $X$ as follows:

$$
d_{r}(X)=a d_{r}(C), \text { for } r \leq L,
$$

where $L:=\max \left\{h^{0}(C, \mathcal{L})-1 \mid \operatorname{deg} \mathcal{L} \leq g-1\right\}$. Here, the gonality sequence $\left(d_{1}(X), \ldots, d_{s}(X)\right)$ of $X$ is defined by the numbers

$$
d_{r}(X):=\min \left\{\operatorname{deg} \mathcal{L} \mid \operatorname{deg} \mathcal{L} \leq g-1, h^{0}(X, \mathcal{L}) \geq r+1\right\} .
$$

\section{KNOWN RESUlts AND EXAMPLES}

In this section, we give some preliminaries and examples to develop and clarify our results.

Assume that $\mathcal{E}$ is a normalized rank 2 vector bundle on a smooth curve $C$ of genus $p$ with $\mathbf{e}=\bigwedge^{2} \mathcal{E}$ and $e:=-\operatorname{deg}(\mathbf{e})$. Let $S=\mathbb{P}(\mathcal{E})$ be an associated ruled surface with a projection morphism $\pi: S \rightarrow C$. We fix a section $C_{0}$ such that $\mathcal{O}_{S}\left(C_{0}\right)=\mathcal{O}_{\mathbb{P}(\mathcal{E})}(1)$. Then, $C_{0}^{2}=-e$. Denote $\mathbf{b} f$ by the pull-back of $\mathbf{b} \in \operatorname{Pic} C$. 
Let $X$ be a smooth irreducible curve of genus $g$ on the ruled surface $\pi: S \rightarrow C$. Then $X$ is numerically equivalent to $a C_{0}+b f$ for some $a, b \in Z$. Note that $X$ becomes an $a$-fold covering of $C$. By the adjunction formula, we have

$$
g=(a-1)\left(b-\frac{1}{2} a e\right)+1+a(p-1),
$$

since the canonical bundle $K_{S}$ is numerically equivalent to $-2 C_{0}+(2 p-2-e) f$.

Note that the irreducibility of $X\left(\neq C_{0}, f\right)$ implies numerical conditions for $a, b$ :

$$
\begin{cases}a>o, \quad b \geq a e & \text { if } e \geq 0 \\ a=1, b \geq 0 \text { or } a \geq 2, b \geq \frac{1}{2} a e & \text { if } e<0 .\end{cases}
$$

In this work, we investigate whether a given line bundle $\mathcal{L}$ on $X$ is composed with the covering morphism $\left.\pi\right|_{X}$. If the covering morphism $\left.\pi\right|_{X}$ is simple, we have the following well-known theorem.

Theorem 2.1 (Castelnuovo-Severi inequality). Let $X$ and $C$ be smooth curves of genus $g$ and $p$. Assume that there exists a covering $f: X \rightarrow C$ of degree $k$ which is a simple morphism. Let $h: X \rightarrow \mathbb{P}^{1}$ be a morphism of degree $d$. If

$$
d<\frac{g-k p+k-1}{k-1},
$$

then the morphism $h$ factors through the covering $f$; that is, there exists a morphism $h^{\prime}: C \rightarrow \mathbb{P}^{1}$ such that $h=h^{\prime} \circ f$.

The main theorem (Theorem 3.10) in this paper gives a sufficient condition for the composedness of a given line bundle $\mathcal{L}$ on $X$ lying on a ruled surface $\pi: S \rightarrow C$, regardless of the simpleness of the covering morphism $\left.\pi\right|_{X}$. Now, we give a couple of examples such that $\left.\pi\right|_{X}$ is not simple.

Example 2.2. Let $X \in \mathbb{P}^{2}$ be a smooth plane curve defined by $x^{2 n}+y^{2 n}=z^{2 n}$. There exists an involution $\eta: X \rightarrow X$ by $(x: y: z) \rightarrow(-x: y: z)$. Then the morphism $\tau: X \rightarrow C$ defined by $(x: y: z) \rightarrow\left(x^{2}: y z: z^{2}\right)$ is a double covering, where $C$ is a plane curve whose defining equation is $x^{n} z^{n}+y^{2 n}=z^{2 n}$. Let $\tilde{C}$ be a normalization of $C$, so we have a natural double covering $\sigma: X \rightarrow \tilde{C}$. (For $n=2$, consult 7], Example 1.1.) A projection from a point $q=(1: 0: 0)$ to the line $\{x=0\}$ is composed with the covering morphism $\sigma$. A blowing-up $\Sigma_{1}$ at $q$ is a ruled surface with a morphism $\pi: \Sigma_{1} \rightarrow \mathbb{P}^{1}$. The restriction $\left.\pi\right|_{X}: X \rightarrow \mathbb{P}^{1}$ is not simple because it factors through $\sigma$.

Example 2.3. Let $C$ be a smooth projective curve genus $p$, and let $\mathcal{L}=\mathcal{O}_{C}(\mathbf{e})$ be a non-trivial line bundle such that $\mathcal{L}^{\otimes 2}=\mathcal{O}_{C}$. Consider a ruled surface $S=$ $\mathbb{P}\left(\mathcal{O}_{C} \oplus \mathcal{O}_{C}(\mathbf{e})\right)$ with a projection $\pi: S \rightarrow C$.

Now take a double covering $\gamma: C^{\prime} \rightarrow C$ with $p^{\prime} \geq 3 p$, where $p^{\prime}$ is a genus of $C^{\prime}$ such that $C^{\prime}$ has a base point free $|\mathbf{b}|$ of degree $p^{\prime}-2 p+1$ such that the divisor $\mathbf{b}$ is not pull-back of a divisor on $C$. The existence of such a double covering is studied in [4] and [2]. Let $\mathcal{E}^{\prime}=\gamma^{*}\left(\mathcal{O}_{C} \oplus \mathcal{O}_{C}(\mathbf{e})\right)$ and $S^{\prime}=\mathbb{P}\left(\mathcal{E}^{\prime}\right)$. Then we have the following diagram:

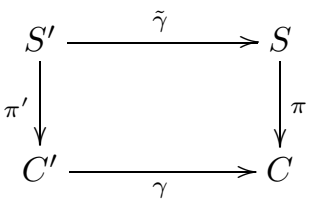


Let $C_{0}^{\prime}$ and $f^{\prime}$ be a minimal section and a fiber on a ruled surface $\pi^{\prime}: S^{\prime} \rightarrow C^{\prime}$. Since $\mathcal{O}_{C^{\prime}}(\mathbf{b})$ and $\mathcal{O}_{C^{\prime}}\left(\mathbf{b}+2 \gamma^{*}(\mathbf{e})\right)\left(\cong \mathcal{O}_{C^{\prime}}(\mathbf{b})\right)$ are globally generated, the linear system $\left|2 C_{0}^{\prime}+\mathbf{b} f^{\prime}\right|$ is base point free by [5], Proposition 36. So, by Bertini's theorem, there exists a smooth curve $X^{\prime}$ on $S^{\prime}$ which is linearly equivalent to $2 C_{0}^{\prime}+\mathbf{b} f^{\prime}$. Since a divisor $\mathbf{b}$ is not a pull-back of a divisor on $C$, the restrict morphism $\left.\tilde{\gamma}\right|_{X^{\prime}}: X^{\prime} \rightarrow S$ is generically one-to-one and the image $X=\left.\tilde{\gamma}\right|_{X^{\prime}}\left(X^{\prime}\right)$ is linearly equivalent to $4 C_{0}+\gamma_{*}(\mathbf{b}) f$. Since the geometric genus $g\left(X^{\prime}\right)=3 p^{\prime}-2 p$ of $X^{\prime}$ is equal to the arithmetic genus $a(X)=3 p^{\prime}-2 p, X$ is a smooth curve on $S$. Clearly, the morphism $\left.\pi\right|_{X}: X \rightarrow C$ factors through the morphism $\left.\pi^{\prime}\right|_{X^{\prime}}: X^{\prime}(\simeq X) \rightarrow C^{\prime}$.

The following notion of extendibility and Serrano's Theorem will be used to prove our main results.

Definition 2.4. Let $X$ be a smooth curve on a smooth surface $S$ and let $\varphi: X \rightarrow$ $X^{\prime}$ be a finite morphism to a smooth curve $X^{\prime}$. Then $\varphi$ is said to be extendible to $S$ if there exists a morphism $\psi: S \rightarrow X^{\prime}$ such that $\left.\psi\right|_{X}=\varphi$. We call $\psi: S \rightarrow X^{\prime}$ an extension of $\varphi: X \rightarrow X^{\prime}$.

Theorem 2.5 (14, Theorem 3.1). Let $X$ be an irreducible smooth curve on a smooth surface $S$. Suppose there exists a morphism $\varphi: X \rightarrow B$ of degree $r$ onto a smooth curve $B$, and let $s \geq 2$ be the degree of some (possibly singular) plane curve birationally isomorphic to $B$. Denote $X^{2}$ the self-intersection number of $X$ in $S$, and set $d=r(s-1)$. If $X^{2}>(d+1)^{2}$ (or $X^{2}>\frac{1}{2}(d+2)^{2}$ and the canonical divisor on $S$ is numerically even), then $\varphi: X \rightarrow B$ is extendible to $S$.

Lemma 2.6 ([1, page 116). Let $C$ be a smooth curve that admits a birational mapping onto a non-degenerate curve of degree $d$ in $\mathbb{P}^{r}$. Then the genus $g$ of $C$ satisfies the inequality

$$
g \leq \pi(d, r):=\frac{m(m-1)}{2}(r-1)+m \epsilon,
$$

where

$$
d-1=m(r-1)+\epsilon, 0 \leq \epsilon \leq r-2 .
$$

Note that the Castelnuovo number $\pi(d, r)$ can be defined as the number regardless of birational embeddings of algebraic curves and has the following property.

Lemma 2.7 ([11, Lemma 6). For $d \geq 3 r-2, r \geq 3$, we have $\pi(d, r) \leq$ $\pi(d-2, r-1)$.

Lemma 2.8 ([10], Lemma 3). Let $\mathcal{M}$ be a base point free line bundle on $X$ with $\operatorname{deg} \mathcal{M} \leq g-1$ such that its associated morphism $\varphi_{\mathcal{M}}$ is birational. If we set $l:=\left[\frac{2 g}{\operatorname{deg} \mathcal{M}-1}\right]$ where $[a]$ denotes the greatest integer less than or equal to a, then

$$
\operatorname{Cliff}(\mathcal{M}) \geq \begin{cases}\frac{g}{3}-1 & \text { for } l=2, \\ \frac{2(l-1)}{(l+1)^{2}} g & \text { for } l \geq 3 .\end{cases}
$$

Remark 2.9. Note that the function $l \rightarrow \frac{2(l-1)}{(l+1)^{2}}$ is decreasing and $\frac{2(l-1)}{(l+1)^{2}} g \leq \frac{g}{3}-1$ for $l \geq 2$. 


\section{MAin Results}

In this section, $S\left(\neq C \times \mathbb{P}^{1}\right)$ is a geometrically ruled surface over a smooth curve $C$ of genus $p$ with invariant $e$. Let $\pi: S \rightarrow C$ be the projection morphism and $C_{0}, f$ denote a minimal section $\left(C_{0}^{2}=-e\right)$, a fiber of the bundle map $\pi: S \rightarrow C$, respectively. Also we denote by $X$ a smooth irreducible curve of genus $g \geq 2$ on $S$ which is numerically equivalent to $a C_{0}+b f(a, b \in Z)$ with $a \geq 2$.

The aim of this paper is to obtain some sufficient conditions for line bundles $\mathcal{L}$ on $X$ to be composed with the covering morphism $\left.\pi\right|_{X}$ in terms of the Clifford index of $\mathcal{L}$. To do this, we first give a range of Clifford index of line bundles on $X$ which are birationally very ample.

Lemma 3.1. Let $\mathcal{L}$ be a birationally very ample line bundle on $X$ with $\operatorname{deg} \mathcal{L} \leq g-1$. If $b>\frac{a e}{2}+1$, then

$$
\operatorname{Cliff}(\mathcal{L}) \geq \begin{cases}\frac{g}{3}-1, & \text { if } a=2 \text { and } m=0, \\ \frac{2(2 a+m-3)}{(2 a+m-1)^{2}} g, & \text { otherwise, }\end{cases}
$$

where $m:=\left[\frac{4 a p}{2 b-a e-2}\right]=\left[\frac{2 a(a-1) p}{g-a p}\right]$.

Proof. Let $d:=\operatorname{deg} \mathcal{L}$. Since $\varphi_{\mathcal{L}}$ is birational, the Castelnuovo-Severi inequality yields $g \leq(a-1)(d-1)+a p$, and so $\left(\frac{g-a p}{g}\right) \frac{2 g}{d-1} \leq 2(a-1)$. The formula $g=$ $(a-1)\left(b-\frac{a e}{2}-1\right)+a p$ gives $g>a p$ for $b>\frac{a e}{2}+1$. Thus

$$
2 \leq\left[\frac{2 g}{d-1}\right] \leq\left[2(a-1)\left(1+\frac{a p}{g-a p}\right)\right] \leq 2(a-1)+\left[\frac{2 a(a-1) p}{g-a p}\right] .
$$

Set $m:=\left[\frac{2 a(a-1) p}{g-a p}\right]$. The result follows from Lemma 2.8 and Remark 2.9 .

Theorem 3.2. Let $\mathcal{L}$ be a birationally very ample line bundle on $X$ with $\operatorname{deg} \mathcal{L} \leq$ $g-1$. Then we get

$$
\operatorname{Cliff}(\mathcal{L}) \geq \begin{cases}\frac{g}{3}-1, & \text { if } a=2 \text { and } b>e+4 p+1, \\ \frac{a-1}{a^{2}} g, & \text { if } b>\frac{a e}{2}+a p+1 .\end{cases}
$$

Proof. If $b>\frac{a e}{2}+a p+1$, we have $m:=\left[\frac{4 a p}{2 b-a e-2}\right]<2$, and so $\operatorname{Cliff}(\mathcal{L})>\frac{a-1}{a^{2}} g-1$ by Lemma 3.1. If $a=2$ and $b>e+4 p+1$, then $m=0$, and hence $\operatorname{Cliff}(\mathcal{L})>\frac{g}{3}-1$ due to Lemma 3.1. Thus the theorem is proved.

Remark 3.3. The conclusion of Theorem 3.2 implies $\operatorname{Cliff}(\mathcal{L})>(a-1) p-1$, since $b>\frac{a e}{2}+a p+1$ is equivalent to $g>a^{2} p$.

Corollary 3.4. Let $\mathcal{L}$ be a birationally very ample line bundle on $X$ with $\operatorname{deg} \mathcal{L} \leq$ $g-1$. Then we have the following:

(i) $\operatorname{Cliff}(\mathcal{L})>2 p-1$ if $a=2$ and $b>e+4 p+1$.

(ii) $\operatorname{Cliff}(\mathcal{L})>\left(a+\frac{a-3}{2 a}\right) p-1$ if $a \geq 3$ and $b \geq \frac{a e}{2}+\frac{a(2 a+1)}{2(a-1)} p+1$.

Proof. If $a=2$ and $b>e+4 p+1$, then we have $g>6 p$, and so $\operatorname{Cliff}(\mathcal{L}) \geq \frac{g}{3}-1>$ $2 p-1$. For $a \geq 3$, the condition $b \geq \frac{a e}{2}+\frac{a(2 a+1)}{2(a-1)} p+1$ yields $g \geq \frac{a(2 a+3)}{2} p$, which gives $\operatorname{Cliff}(\mathcal{L})>\frac{a-1}{a^{2}} g-1 \geq\left(a+\frac{a-3}{2 a}\right) p-1$.

Next, we will deal with the line bundles which are not birationally very ample. 
Theorem 3.5. Assume $e \geq 0$ and $S \neq C \times \mathbb{P}^{1}$. Let $\mathcal{L}$ be a base point free line bundle on $X$ with $\operatorname{Cliff}(\mathcal{L})<b-\frac{a e}{2}-2$. If $\varphi_{\mathcal{L}}: X \rightarrow \varphi_{\mathcal{L}}(X) \subset \mathbb{P}^{r}\left(:=\mathbb{P} H^{0}(X, \mathcal{L})\right)$ is extendible to $\psi: S \rightarrow \varphi_{\mathcal{L}}(X) \subset \mathbb{P}^{r}$, then $\mathcal{L}$ is composed with the covering morphism $\left.\pi\right|_{X}: X \rightarrow C$.

Proof. Set $X^{\prime}:=\varphi_{\mathcal{L}}(X)$. Let $D=\alpha C_{0}+\xi f$ be a pull-back of a hyperplane section $H$ of $X^{\prime}$ in $\mathbb{P}^{r}$ via the morphism $\psi: S \rightarrow X^{\prime} \subset \mathbb{P}^{r}$. Then, we have the following three inequalities:

$$
\text { (1) } f . D \geq 0 ; \quad \text { (2) } C_{0} . D \geq 0 ; \quad \text { (3) } D^{2} \equiv 0 .
$$

This gives the following numerical criteria:

$$
\text { (1) } \alpha \geq 0 ; \quad(2) \beta \geq \alpha e ; \quad(3) \alpha(2 \beta-\alpha e)=0,
$$

where $\beta:=\operatorname{deg} \xi$. Assume that $\alpha>0$. Let $f$ be a smooth fiber of $\pi$. Consider the morphism $\left.\psi\right|_{f}: f\left(\cong \mathbb{P}^{1}\right) \rightarrow X^{\prime} \subset \mathbb{P}^{r}$, which is associated with the line bundle $\mathcal{O}_{S}(D) \otimes \mathcal{O}_{f}$. Then it is not a constant morphism since $\operatorname{deg} \mathcal{O}_{S}(D) \otimes \mathcal{O}_{f}=f . D=$ $\alpha>0$, so the image curve $\left.\psi\right|_{f}(f)$ is rational, i.e., $X^{\prime} \simeq \mathbb{P}^{1}$. Since $X^{\prime}$ is nondegenerate, we have $\alpha \geq r$ by the Riemann-Roch Theorem.

Now, consider the Clifford index of $\mathcal{L}$ :

$$
\operatorname{Cliff}(\mathcal{L})=\operatorname{deg} \mathcal{L}-2 r=\mathcal{O}_{S}(D) . X-2 r=-a \alpha e+a \beta+\alpha b-2 r \geq \alpha\left(b-\frac{a e}{2}-2\right),
$$

since $r \leq \alpha$ and $2 \beta=\alpha e$. This cannot happen under the assumption $\operatorname{Cliff}(\mathcal{L})<$ $\left(b-\frac{a e}{2}-2\right)$.

Consequently, we have $\alpha=0$, whence $D=\xi f$. It means that $\mathcal{L}$ is composed with the covering morphism $\left.\pi\right|_{X}: X \rightarrow C$. Thus the theorem is proved.

Theorem 3.6. Let $\mathcal{L}$ be a base point free line bundle on $X$ whose associated morphism $\varphi_{\mathcal{L}}$ is neither birational nor extendible to $S$. Then

$$
\operatorname{Cliff}(\mathcal{L}) \geq \sqrt{a(2 b-a e)}-3,(\operatorname{Cliff}(\mathcal{L}) \geq \sqrt{2 a(2 b-a e)}-4, \quad \text { in case e is even }) .
$$

Proof. Let $\varphi_{\mathcal{L}}$ be the morphism associated to the line bundle $\mathcal{L}$. Set $\mu:=\operatorname{Cliff}(\mathcal{L})$. Assume that $\operatorname{dim}|\mathcal{L}|=1$. Since the morphism $\varphi_{\mathcal{L}}$ is not extendible to $S$, by Theorem 2.5 we have

$$
\begin{gathered}
a(2 b-a e)=X^{2} \leq(\mu+3)^{2}, \\
\left(a(2 b-a e)=X^{2} \leq \frac{(\mu+4)^{2}}{2}, \text { in case } e \text { is even }\right) .
\end{gathered}
$$

Now we assume that $\operatorname{dim}|\mathcal{L}| \geq 2$. Set

$$
m:=\operatorname{deg} \varphi_{\mathcal{L}}, X^{\prime}:=\varphi_{\mathcal{L}}(X) \text { and } d:=\operatorname{deg} \mathcal{L} .
$$

Since $\varphi_{\mathcal{L}}$ is not birational, $m \geq 2$. Let $\sigma: \widetilde{X^{\prime}} \rightarrow X^{\prime}$ be a normalization of $X^{\prime}$. Then we have the morphism $\widetilde{\varphi_{\mathcal{L}}}: X \rightarrow \widetilde{X^{\prime}}$ satisfying $\sigma \circ \widetilde{\varphi_{\mathcal{L}}}=\varphi_{\mathcal{L}}$. If $\widetilde{\varphi_{\mathcal{L}}}$ is extendible 
to $S$, then we have the following commutative diagram:

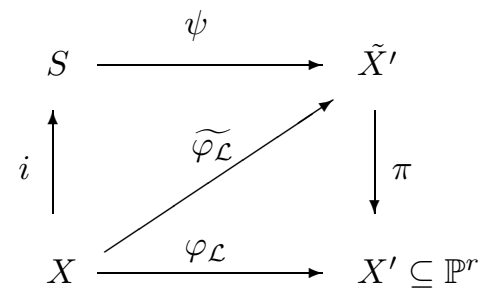

It implies the extendibility of $\varphi_{\mathcal{L}}$, which is a contradiction to the assumption. Thus $\widetilde{\varphi_{\mathcal{L}}}$ is also not extendible.

Now, consider a projection of $X^{\prime}$ from general $(r-2)$-points $\sum_{i=1}^{r-2} Q_{i}$ of $X^{\prime}$, which gives a plane model of $\widetilde{X^{\prime}}$ with degree $\frac{d}{m}-r+2$. Due to Theorem 2.5.

$$
a(2 b-a e)=X^{2} \leq\left\{m\left(\frac{d}{m}-r+1\right)+1\right\}^{2} \leq(\mu+3)^{2},
$$

since $m\left(\frac{d}{m}-r+1\right)=d-m r+m \leq d-2 r+2=\mu+2$. Moreover, if $e$ is even, Theorem 2.5 yields

$$
a(2 b-a e)=X^{2} \leq \frac{1}{2}\left\{m\left(\frac{d}{m}-r+1\right)+2\right\}^{2} \leq \frac{(\mu+4)^{2}}{2} .
$$

Thus the result follows.

Now, we can reprove weak versions of Martens' theorem ([12, Theorem) and Harui's theorem ([6], Theorem 2.4) using Theorem 3.6.

Theorem 3.7 (G. Martens). Let $\Sigma_{e}$ be a rational ruled surface with invariant $e$ and let $X \in\left|a C_{0}+b f\right|$ be an irreducible smooth curve on a surface $\Sigma_{e}$ with $a \geq 2$. If $e \geq 2$, then $\operatorname{gon}(X)=a$ and $\operatorname{Cliff}(X)=a-2$.

Proof. Let $\mathcal{L}$ be a line bundle computing the gonality of $X$ and assume that $\operatorname{deg} \mathcal{L}<$ $a$. Since we assume $e \geq 2$ and the irreducibility of $X$ implies $b \geq a e$, we have

$$
\operatorname{Cliff}(\mathcal{L}) \leq a-3<b-\frac{a e}{2}-2 \quad \text { and } \quad \operatorname{Cliff}(\mathcal{L}) \leq a-3<\sqrt{a(2 b-a e)}-3 .
$$

Therefore the morphism $\varphi_{\mathcal{L}}$ is extendible to $\Sigma_{e}$ by Theorem 3.6, and so composed with the covering morphism by Theorem 3.5. It gives a contradiction to the assumption $\operatorname{deg} \mathcal{L}<a$. Consequently, we have $\operatorname{gon}(X)=a$.

Now, we prove the result for the Clifford index. If $a=2$, it is trivial. Assume $c:=\operatorname{Cliff}(X)<a-2$ and $a \geq 3$. Let

$$
r:=\min \left\{h^{0}(X, \mathcal{M})-1 \mid \operatorname{deg} \mathcal{M} \leq g-1, h^{0}(X, \mathcal{M}) \geq 2, \operatorname{Cliff}(\mathcal{M})=c\right\} .
$$

Then we obtain $r \geq 2$, since $\operatorname{gon}(X)=a$ and $\operatorname{Cliff}(X)<a-2$. Let $\mathcal{L}$ be a line bundle computing the Clifford index of $X$ with $h^{0}(X, \mathcal{L})=r+1$. The minimality of $r$ implies that $\mathcal{L}$ is very ample. If $r=2, X$ is a smooth plane curve of degree $a+1$ since $\operatorname{gon}(X)=a$. Then, we have $g(X)=\frac{a(a-1)}{2}$. On the other hand, since $X$ is a smooth curve on $\Sigma_{e}$ with $e \geq 2$,

$$
g(X)=(a-1)\left(b-\frac{a e}{2}\right)-a+1 \geq a(a-1)-a+1,
$$


which is a contradiction to $a \geq 3$. Now we assume that $r \geq 3$. Lemma 2.7 yields

$$
a^{2}-2 a+1 \leq g \leq \pi(2 a+3,3) \leq \frac{a^{2}+2 a}{4}
$$

which cannot occur for $a \geq 3$. Thus the theorem is proved.

Remark 3.8. In Theorem 3.7 the result on the Clifford index of $X$ is added to Martens' original theorem.

Theorem 3.9 (Harui). Let $S$ be a geometrically ruled surface over an elliptic curve $E$ and let $X \in\left|a C_{0}+b f\right|$ be an irreducible smooth curve on $S$. If $e \geq 4$, then $\operatorname{gon}(X)=2 a$ and $\operatorname{Cliff}(X)=2 a-2$.

Proof. First, we prove the results for the gonality. Let $\mathcal{L}$ be a line bundle computing the gonality of $X$ and assume that $\operatorname{deg} \mathcal{L}<2 a$. As in the proof of Theorem 3.7, we have

and

$$
\operatorname{Cliff}(\mathcal{L}) \leq 2 a-3<b-\frac{a e}{2}-2
$$

$$
\operatorname{Cliff}(\mathcal{L}) \leq 2 a-3< \begin{cases}\sqrt{a(2 b-a e)}-3, & \text { for } e \geq 5 \\ \sqrt{2 a(2 b-a e)}-4, & \text { for } e=4\end{cases}
$$

which yield gon $(X)=2 a$.

For the Clifford index of $X$, as in the proof of Theorem 3.7, we assume that $c<2 a-2$ and $r \geq 2$. If $r=2, X$ is a smooth plane curve of degree $2 a+1$ since gon $(X)=2 a$. However, the line bundle computing the gonality of a smooth plane curve is associated to the projection from a point on $X$, and hence it is not composed with the covering morphism. So we have $r \geq 3$. Lemma 2.7 yields

$$
g \leq \pi(2 a+3,3)=a^{2}+a,
$$

from which we get $a=2$ since $g \geq(a-1)\left(\frac{a e}{2}\right)+1 \geq 2 a^{2}-2 a+1$. It cannot occur, since the Clifford index of a bielliptic curve is equal to 2 . Thus we have $\operatorname{Cliff}(X)=2 a-2$.

Now combining Corollary 3.4. Theorem 3.5, and Theorem 3.6, we obtain the following theorem.

Theorem 3.10. Assume $b>\frac{a e}{2}+\delta$, where

$$
\delta:= \begin{cases}\max \left\{4 p+1,(p+1)^{2}\right\}, & \text { for } a=2, \\ \max \left\{\frac{a(2 a+1) p}{2(a-1)}+1, \frac{a(p+1)^{2}}{2}\right\}, & \text { for } a \geq 3 .\end{cases}
$$

Let $\mathcal{L}$ be a line bundle on $X$ with $h^{0}(X, \mathcal{L}) \geq 2$ and let $\operatorname{deg} \mathcal{L} \leq g-1$. If $\operatorname{Cliff}(\mathcal{L})<$ ap -1 , then the base point free part of $\mathcal{L}$ is composed with the covering morphism $\left.\pi\right|_{X}: X \rightarrow C$.

Proof. We may assume $\mathcal{L}$ is base point free. Corollary 3.4 implies that $\mathcal{L}$ is not birationally very ample for $\operatorname{Cliff}(\mathcal{L})<a p-1$ and $b>\frac{a e}{2}+\delta$. By Theorem 3.6. $\varphi_{\mathcal{L}}$ is extendible since we have $\operatorname{Cliff}(\mathcal{L})<a p-1 \leq \sqrt{a(2 b-a e)}-3$ for $b>\frac{a e}{2}+$ $\frac{a(p+1)^{2}}{2}$. Consequently, $\mathcal{L}$ is composed with the covering morphism $\left.\pi\right|_{X}: X \rightarrow C$ by Theorem 3.5 . Thus the theorem is proved.

From Theorem 3.10 we obtain the following result on the gonality sequence of $X$. 
Corollary 3.11. Under the same assumption as in Theorem 3.10, we have

$$
d_{r}(X)=a d_{r}(C) \text { for } r \leq L,
$$

where $\left(d_{1}(C), \ldots, d_{L}(C)\right)$ and $\left(d_{1}(X), \ldots, d_{s}(X)\right)$ denote the gonality sequences of $X$ and $C$ and where $L$ is the length of the gonality sequence of $C$, i.e., $L:=$ $\max \left\{h^{0}(C, \mathcal{L})-1 \mid \operatorname{deg} \mathcal{L} \leq g-1\right\}$. Specifically, if $C$ is a general $k$-gonal curve of genus $p$, then

$$
d_{r}(X)=\text { ark, for } r \leq \frac{p-4}{2(k-2)} .
$$

Proof. The former part trivially holds by Theorem 3.10, The latter part follows from Theorem 3.1 in $\left[9\right.$, which shows the equality $d_{r}(C)=r k$ for $r \leq \frac{p-4}{2(k-2)}$ on a general $k$-gonal curve $C$.

Remark 3.12. If $\left.\pi\right|_{X}: X \rightarrow C$ is simple, the conclusion of Theorem 3.10 trivially holds by the Castelnuvo-Severi inequality (Theorem 2.1). However, the CastelnuvoSeveri inequality cannot be applied to the general cases where the covering morphisms $\left.\pi\right|_{X}$ are not simple. In such cases, one may attempt to decompose $\left.\pi\right|_{X}$ by

$$
\left.\pi\right|_{X}: X=X_{0} \stackrel{\phi_{1}}{\longrightarrow} X_{1} \stackrel{\phi_{2}}{\longrightarrow} \cdots \stackrel{\phi_{s-1}}{\longrightarrow} X_{s-1} \stackrel{\phi_{s}}{\longrightarrow} X_{s}=C
$$

where the $\phi_{i}$ 's are simple for $i=1, \ldots, s$, and apply the Castelnuvo-Severi inequality to each simple $\phi_{i}: X_{i-1} \rightarrow X_{i}$. However, this kind of approach is not effective if one of $\phi_{1}, \ldots, \phi_{s}$ has a small ramification index as follows.

Assume $\mathcal{L}=\phi_{1}^{*} \mathcal{L}_{1}, \mathcal{L}_{1}=\phi_{2}^{*} \mathcal{L}_{2}, \ldots, \mathcal{L}_{t-1}=\phi_{t}^{*} \mathcal{L}_{t}$ for some $\mathcal{L}_{i}$ on $X_{i}$ by applying the Castelnuovo-Severi inequality to $\phi_{i}$ for $i \leq t<s$. Set $k:=\operatorname{deg} \phi_{t+1}, q:=$ $g\left(X_{t}\right), \gamma:=g\left(X_{t+1}\right)$, and $R:=$ the ramification divisor of $\phi_{t+1}$. If

$$
\operatorname{deg} R<2(k-1) \frac{\operatorname{deg} \mathcal{L}}{a_{1} \cdot a_{2} \cdots a_{t}}, \quad a_{i}:=\operatorname{deg} \phi_{i},
$$

then we have $\operatorname{deg} \mathcal{L}_{t}=\frac{\operatorname{deg} \mathcal{L}}{a_{1} \cdot a_{2} \cdots a_{t}}>\frac{q-k \gamma+k-1}{k-1}$, since $\operatorname{deg} R=2(q-k \gamma+k-1)$ by the Riemann-Hurwitz formula. Then the Castelnuvo-Severi inequality is not available for $\mathcal{L}_{t}$. As a result, such a step-by-step approach is not adaptable in the case where one of the $\phi_{i}$ 's has so small a degree of ramification divisor.

\section{REFERENCES}

[1] Arbarello, E., Cornalba, M., Griffiths P. A. and Harris J., Geometry of Algebraic Curves. I, Springer Verlag, 1985. MR.770932 (86h:14019)

[2] Ballico, E., Keem, C. and Park, S., Double covering of curves, Proc. Amer. Math. Soc. 132 (11) (2004) 3153-3158. MR2073288 (2005a:14037)

[3] Coppens, M. and Martens, G., Secant spaces and Clifford's theorem, Compositio Math. 78 (1991) 193-212. MR.1104787 (92e:14021)

[4] Fuentes García, L., Pencils on double coverings of curves, Arch. Math. 92 (2009) 35-43. MR2471986 (2010d:14051)

[5] Fuentes García, L. and Pedreira, M., The projective theory of ruled surfaces, Note Mat. 24 (1) (2004/2005) 25-63. MR2199622 (2006k:14065)

[6] Harui, T., The gonality and the Clifford index of curves on an elliptic ruled surface, Arch. Math. 84 (2005) 131-147. MR2120707 (2006b:14053)

[7] Harui, T., Komeda, J. and Ohbuchi, A., Double coverings between smooth plane curves, Kodai Math. J. 31 (2) (2008) 257-262. MR2435894(2009j:14035)

[8] Keem, C. and Kim, S., On the Clifford index of a general $(e+2)$-gonal curve, Manuscripta Math. 63 (1989) 83-88. MR975470 (89m:14016) 
[9] Kim, S., On the Clifford sequence of a general $k$-gonal curve, Indag. Math. (N.S.) 8 (1997) 209-216. MR1621995 (99d:14006)

[10] Kim, S., Normal generation of line bundles on multiple coverings, J. Algebra 323 (9) (2010), 2337-2352. MR 2602382

[11] Kim, S. and Kim, Y., Projectively normal embedding of $k$-gonal curve, Comm. Algebra 32 (1) (2004) 187-201. MR2036230 (2005b:14066)

[12] Martens, H., The gonality of curves on a Hirzeburch surface, Arch. Math. 67 (1996) 349-352. MR:1407339 (97k:14036)

[13] Meis, T., Die minimale Blätterzahl der Konkretisierungen einer kompakten Riemannschen Fläsche. Schriftenreishe des Math. Inst. d. Univ. Münster 16 (1960). MR0147643 (26:5158)

[14] Serrano, F., The adjunction mapping and hyperelliptic divisors on a surface, J. Reine Angew. Math. 381 (1987) 90-109. MR918842 (89c:14054)

[15] Shin, D., Base point free pencils on triple covers of smooth curves, Int. J. Math. 19 (6) (2008) 671-697. MR2431633 (2010e:14025)

Department of Mathematics Education, Yeungnam University, 214-1 Daedong Gyeongsan, 712-749, Gyeongsangbuk-do, Republic of Korea

E-mail address: ychoi824@yu.ac.kr

Department of Electronics, Chungwoon University, Hongseong, Chungnam, 350-701, REPUBLIC OF KoREA

E-mail address: sjkim@chungwoon.ac.kr 